\title{
System of Mediation based on Ontology to Solve the Semantic Heterogeneity between Information Systems
}

\author{
Gaou Salma \\ Faculty Science. University \\ Abdelmalek Essaadi \\ BC Jbel Hayane Oulianti Lot 7 \\ N 3 BP 565 ETG. Tetouan, \\ MOROCCO
}

\author{
Stouti EL Mehdi \\ Faculty Science. University \\ Abdelmalek Essaadi \\ Hay aghras av razi etage 3 appt \\ 4 Martil, MOROCCO
}

\author{
El Kadiri Kamal Eddine \\ Faculty Science. University \\ Abdelmalek Essaadi, \\ Mhannech II B.P: 2121. \\ Tetouan, MOROCCO
}

\begin{abstract}
The Semantic Web is a new vision of the Web, where resources are described by meta-data, using the conceptual vocabulary provided by the ontology, which allow software agents to better assist users in their various resources access Web.

E-learning is considered as one of the areas in which the Semantic Web can make real progress that is looking for information or reuse of learning resources, even in customizing monitoring training.

In this article, we focus on the semantic heterogeneity between information systems and we propose a mediation system based on ontologies, to allow the integration of heterogeneous sources. Ontologies allow systems to use a terminology consensus. We use the mapping between different ontologies. This mapping will be used in the process of query rewriting. Ontologies and the mapping are described with OWL. we present our proposed method for translating SPARQL queries in XQuery queries. This method is based on the XML-to-ontology mapping.
\end{abstract}

\section{Keywords}

OWL System, mediation, XML, Ontology, semantic Web.

\section{INTRODUCTION}

At its creation by Tim Berners Lee in the early 90s, the goal of the Web was to allow any user to access and share large amounts of information on the net. HTML was a very good solution for formatting quick and easy online document, after this innovation the Web has achieved its objectives.

In this paper, we will present some mapping approaches that have emerged in recent years.

We will do a comparison or evaluation approaches to visualize their changes, and to see from the mapping techniques presented above, those are the most used.

- PROMPT [1] (Stanford Medical Informatics) is originally developed to support the merging of ontologies. It can store the mappings between ontologies sources created by the system and the user. The discovery of mappings based on lexical features (terminological methods) and structural (structural methods), as well as suggestions from the user during the merger process.

- $\quad$ FCA-Merge (Formal Concept Analysis - Merge) [2] (University of Karlsruhe) compares two ontologies that have a set of shared instances or a set of annotated documents. Based on formal analysis techniques concepts (and extensional structural and technical), this approach can support the equivalence as well as links specialization. The results of this method can be used by a knowledge engineer to perform merging ontologies.

- IF-Map (Information-Flow-based Map) [3] (University of Southampton and University of Edinburgh) to automatically identify the mappings based on the theory of information flow by exploiting structural and extensional techniques. IF-Map can generate from two ontologies logical isomorphism (ontology mapping), which is then translated into mappings using the theory of flow.

GLUE [27] (University of Washington) is an example of an approach that uses learning techniques to find mappings. GLUE uses multiple learners to exploit the information in the bodies and the taxonomy of the ontology. It then uses a probabilistic model to combine the results of different learners.

- QOM (Quick Ontology Mapping) [28] [29] (University of Karlsruhe), based on the idea that the loss of quality of the mapping is marginal similarity measures that are used and produced by a mapping base are refined the sigmoid function, it consists of the weighted results, and finally as a threshold average function, the final mapping is produced.

- RiMOM (Risk Minimization based Ontology Mapping) [30] (Tsinghua University) it makes the mapping of ontologies as a problem of decision making, finding the best mapping is to find the decision with minimal risk, it allows the discovery complex mappings, mappings between concepts and mappings between roles and uses statistical techniques and extensional.

- CTXMatch [31] [32] (University of Trento and ITCIRST) uses semantic techniques can transform the problem of mapping a logical problem is validation, as it calculates the logical equivalence relations, subsumption between concepts and properties.

- In this article, our approach is based, we focus on the semantic heterogeneity between information and we propose a mediation system based on ontology systems to enable the integration of heterogeneous and distributed sources. Ontologies allow systems to use a consensus terminology. We use the Global As View (GAV) approach for mapping between different ontologies. This mapping will be used in the process of rewriting queries. Ontologies and the mapping are described with OWL. We use XQuery to query 
the XML documents as query language language at our mediator and an algebra for XML query processing.

\section{ONTOLOGIES}

The word ontology is used in different contexts. Its origin is attributed to the field of philosophy. It is a branch of metaphysics and means the science or theory of being. In the context of computer science, the term ontology appeared in the field of Artificial Intelligence (AI) recently.

Ontology has been several definitions in the field of Artificial Intelligence, from which we quote the definition given by Gruber: "An ontology is an explicit specification of a conceptualization" [6]. The term is described by Gruber conceptualization as an abstract view, simplified world that we wish to represent for some purpose. The term explicit means that the type of concepts used and the constraints on their use are explicitly defined.

Depending on the purpose of conceptualization, [7] distinguishes four types of ontologies.

- The domain ontologies: they have a vocabulary and concepts describing an application domain.

- $\quad$ The application ontologies: they are more specific as they have a limited vocabulary and used for a given application.

- The generic ontologies: ontologies or high-level ontologies were expressing valuable knowledge in many areas, they treat classes of things or concepts of high abstraction such as entities, events or relations.

- The representation of ontologies: ontologies or meta, they conceptualize the primitive language of knowledge representation, such as a Frame integrates ontology representation languages primitives based frames such as classes, instances, properties, etc. .

Ontologies are formal and consensual specifications of conceptualizations that provide a shared understanding of a domain, an understanding that can be communicated across people and application systems [8]. The field of ontology draws attention because it provides: 1) a basic conceptual structure from which it is possible to develop knowledgebased systems that are sharable and reusable, 2) an interoperability between information sources and knowledge. It is necessary to consider the difference between two types of ontologies: an ontology-oriented Semantic Web and ontologyoriented concept. An ontology oriented concept addresses the fundamental concepts of the target world that need to be examined in depth while an ontology oriented Semantic Web is a computer-readable vocabulary which defines the meaning of metadata and is used primarily to achieve semantic interoperability between information resources through metadata. Such ontologies can be described as ontology surface, since it does not necessarily deep conceptual structure of the target world. The engineering knowledge (IC) has long been considered the favorite field of development of expertise in system design based on knowledge [6]. Historically, ontological engineering (IO) emerged from the knowledge engineering, ontological engineering to specify the conceptualization of a system, to provide a formal representation of knowledge to be acquired in the form of declarative knowledge exploitable by an agent [5]. Thus, the operating mechanism by inference, a type representation as declarative ontology, while following the inference rules defined in this ontology, is the source of the Intelligence system. The knowledge engineering has given rise to ontological engineering, where the ontology is the key object which must be addressed. The need for ontology and ontological engineering of knowledge-based systems beginning to be understood and accepted [9].OWL ontologies are used to model domain knowledge [10].

\section{THE SEMANTIC WEB AND APPLICATION AREAS}

\subsection{Information retrieval module}

The Semantic Web seeks to achieve a mastery of content, to provide relevant answers to users. For example, a teacher looking for a document type "race" will certainly be an appropriate response from the moment he can express it seeks a document type "race" is that every document has a metadata "type of document "that comes from the ontology that describes the documents pedagogical this is not the case if the application was run on a conventional search engine, which will return documents containing the word" race "and those who contain the word "of course."

The search for information is part of most of the Web applications include: e-learning, e-commerce, etc...

\subsection{Adaptation / module rewrite the query}

Through the Internet, a potentially infinite number of services and documentation accessible to all users. Most of the services and materials provided on the Internet today offer an organization, content, mode of interaction and unique presentation for all. This may be sufficient in some cases. But not all users are not interested in the same information and have the same expectations, knowledge, skills, interests, etc... [13]

This is the case in an e-learning system, we try to adapt learning content to the learner preferences, and give teachers the opportunity to reuse content, to combine with one another to build a new document.

All this may be possible if the content and structure of documents used for training, are described through ontology and if we can have standard models user / use the form of ontology.

\subsection{Integration of heterogeneous data sources module}

The goal of integrating heterogeneous data sources is to provide users with a consistent view of the system that uses them. E-commerce has a very good example of areas that can benefit from such an improvement.

Before, the user was required to ask itself the source of data, each with its query language, the work has been done in the context of Web to build homogeneous systems based on one of two approaches: A mediator approach and data warehouses.

The Semantic Web approach reuses the mediator, while adding an ontology will provide a common vocabulary that will be used to query the system.

A mediator will query data sources, and respond to the request initiated by the user, also using terms of the ontology.

\subsection{Heterogeneity}

We consider the heterogeneity of representation that involves modeling constructs for data relevant to the structural 
interoperability. Schematic heterogeneity appears especially in structured databases is also an aspect of the structural heterogeneity. Although significant progress has been made in the system, the syntactic interoperability and structural / schematic, comprehensive solutions to semantic interoperability remain [23], [24]. However, several trends and advances in software technology continue to draw attention to semantic issues and semantic interoperability. This is the subject of this special section.

Can be distinguished heterogeneity of information systems and interoperability concerns related:

$\checkmark \quad$ heterogeneity of information

- $\quad$ semantic heterogeneity

- Structural representation / Diagram heterogeneous.

- $\quad$ The syntactic heterogeneity Format

- $\quad$ The heterogeneity of the system

$\checkmark \quad$ Heterogeneity information system

- Digital Media Systems Management Repository

- Database management systems (DBMS heterogeneity, data models, system functionality such as concurrency control and recovery)

\section{$\checkmark \quad$ Heterogeneity platform}

- Operating systems (het. file system, names, file types, operation, transaction support, IPC)
- Equipment / system (heterogeneous set of instructions, data representation / coding)

$\begin{array}{ll}\checkmark & \text { Semantic interoperability } \\ \checkmark & \text { Structural interoperability } \\ \checkmark & \text { Syntactic interoperability } \\ \checkmark & \text { Interoperability system[25]. }\end{array}$

\subsection{Global Ontology}

This ontology will provide an overview of all the attributes used to describe the data. It is used to define the mappings (correspondences) between the concepts through equivalence relations[27].

\subsection{Local Ontology}

The local ontology must be linked to the real information. Therefore, the mappings must be provided between the local ontology and each source of information[27].

\subsection{Mapping Web Service}

Mapping Web Service is a specification of semantic overlap between two ontologies is the output of the mapping process. Correspondence between the different entities of the two ontologies is usually expressed using the axioms formulated in a language specific mapping. The three main phases of any mapping process are: (1) mapping discovery, (2) mapping representation, and(3) mapping exploitation/execution[22].

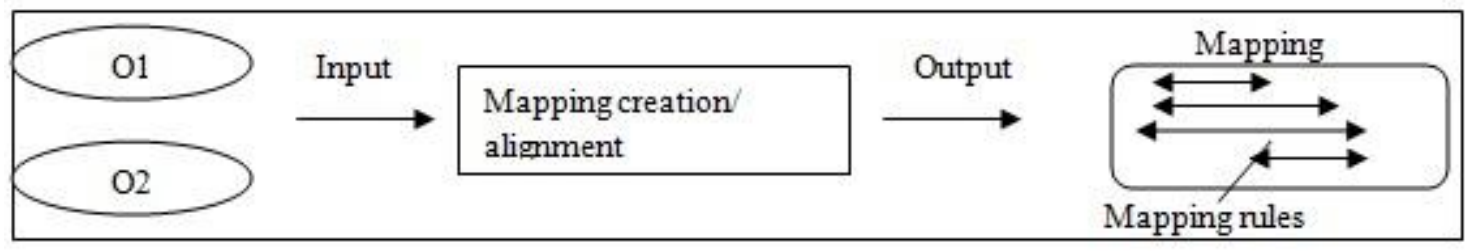

Fig 1: Ontology mapping

\subsection{Querying Web Service:}

Once the various data providers are connected to the module knowledge base, users can query using the Querying Web Service. When a query (expressed in SPARQL [26]) is submitted to the system, it is analyzed by this service and decomposed into a set of sub-queries.

Then, using the directory mapping in the knowledge base, the web service redirects the request subqueries data providers appropriate[28]. 


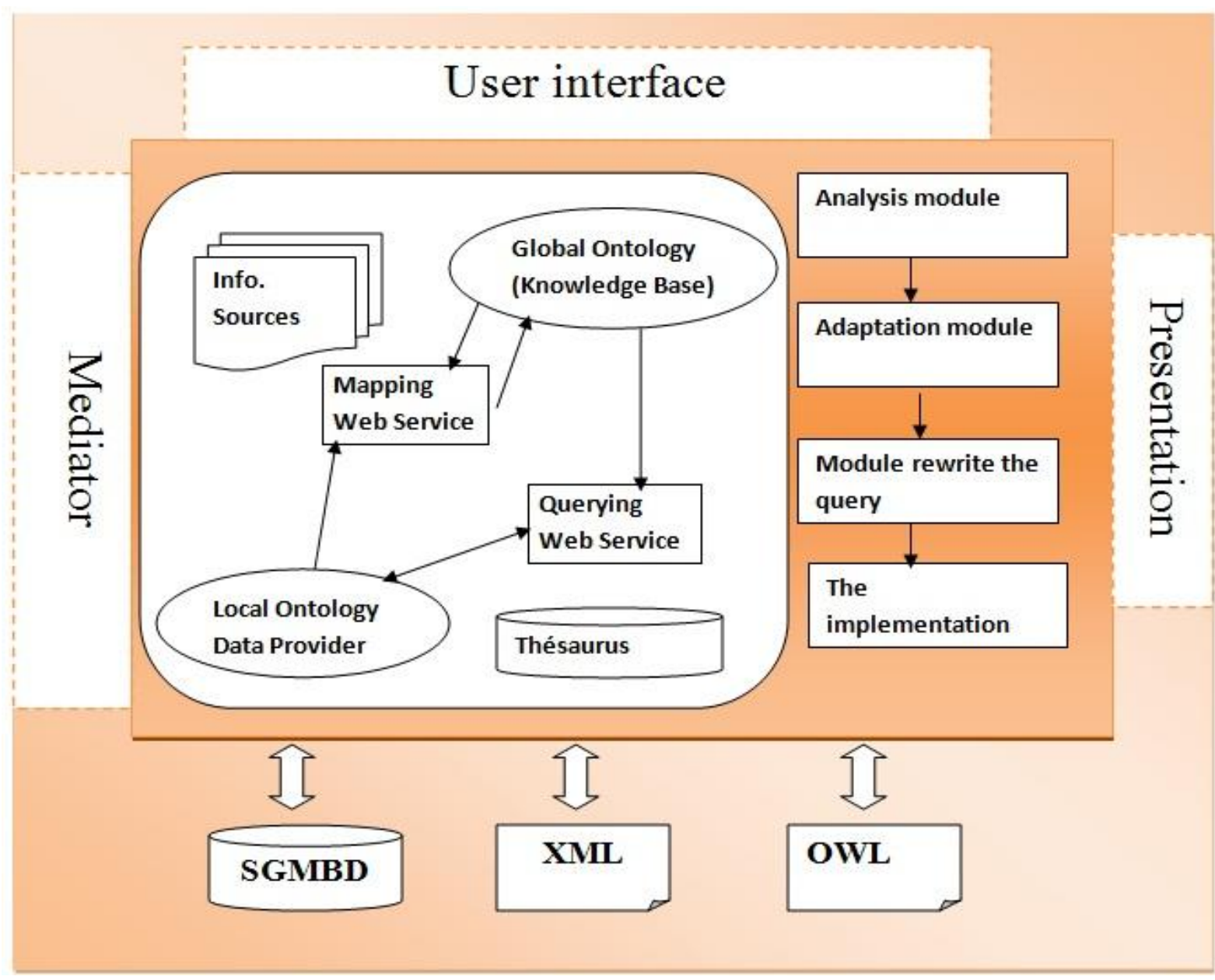

Fig 2. System Architecture of system mediation

Our mediation system has three levels: the interface, the mediator level, the level of local sources in the following, we briefly describe the mediator and the adapter and the general treatment of a query.

This approach consists of three basic steps. The first step is seeking candidates concepts depending on the context, it can enrich the semantics of concepts in the context, and then it performs a comparison between these contexts to select the closest concepts. The second step involves several search strategies and allows for multiple operations similarity computation

It uses four strategies for calculating similarity, the similarity of description, neighborhood, taxonomies and instances. Our approach exploits the majority of mapping techniques, among others, similarity between contexts (semantic techniques), the similarities of description, neighborhood and taxonomy (corresponding to intentional techniques), and the similarities of instances (meaning extensional techniques). After This mapping will be used in the process of rewriting queries. Ontologies and the mapping are described with OWL. We use XQuery to query the XML documents as query language language at our mediator and an algebra for XML query processing.

\section{TECHNICAL STUDY}

The language we chose to represent knowledge in Wasit is OWL. The latter consists of three sublanguages increasing expressiveness: OWL Lite, OWL DL and OWL Full. In our system of mediation, we used OWL DL and OWL Lite. It is sufficient to represent the thesaurus as it allows expressing hierarchies and simple constraints. As for OWL DL, it contains all the language constructs but with restrictions on the hierarchy. It has more the expressive power of description logics (Description Logic). We chose OWL DL ontologies to represent because it is expressive enough to describe the mapping between ontologies and it ensures completeness (all inferences are computable) and decidable reasoning mechanisms (all computations terminate in a finite time).

\subsection{OWL}

OWL (Web Ontology Language) was designed to be used by applications that process the content of information instead of just presenting it to humans. OWL greatly facilitates interoperability at the machine level of Web content by providing additional vocabulary along with formal semantics. OWL sub-languages have more expressive OWL Lite, OWL DL and OWL Full.

The W3C OWL has three sublanguages with increasing capacity of expression and it is as necessary that we choose the appropriate language.

The W3C OWL has three sublanguages with increasing capacity of expression and it is as necessary that we choose the appropriate language. 
OWL is based on RDF (Resource Description Framework) and RDFS (Resource Description Framework Schema) [14]. $\mathrm{RDF}$ is a language for describing resources and their relationships while RDFS provides a vocabulary for describing classes of RDF resources with type generalization hierarchies of classes and properties.

OWL is the successor of DAML + OIL [15]. It is a declarative language, formally defined. It is used to represent concepts (owl: class), relations between concepts (owl: ObjectProperty), data types (owl: DatatypeProperty) and also cardinalities. In addition, OWL is used to characterize the relationship as transitivity (owl: TransitiveProperty) and symmetry (owl: SymetricProperty). It supports hierarchies of specialization / generalization (rdfs: subClassOf). Equivalent concepts can be connected (owl: equivalentClass or owl: sameClass). The union and intersection of classes is possible (owl: unionOf, owl: intersectionOf).

An OWL ontology can be represented in different formats: NTriple, or abstract syntax RDFS serialization / XML. [4]

\subsection{Choice of the algebra XML}

It is from this observation that the $\mathrm{W} 3 \mathrm{C}$ has developed the XML standard, XML is a W3C Recommendation since February 10, 1998, and this language is a metalanguage to facilitate the development of specialized markup languages.

We chose to use XML algebra for modeling formally queries on unstructured data. There are several works on algebra XML [16] [17]. The XAT algebra [18], we adopted for query processing in Wasit, was developed by Niagara [19] and adapted by Xear [20].

The XAT algebra is sufficiently powerful and complete account of all the characteristics of semi-structured data, including navigation and formatting. It has several operators. Some are specific to XML format and as follow, others are from the relational algebra as select, Cartesian product, union, etc..

Some operators are unary (follow, source, etc..), Others are binary (union, join, etc.).. [4]

\subsection{Diagrams}

The detailed design phase of the system is the next stage of our development process. This phase is to take the analysis model, and again depending on the architecture adopted:

\subsubsection{Use case diagram}

The figure below shows the interaction between system users and system use cases:

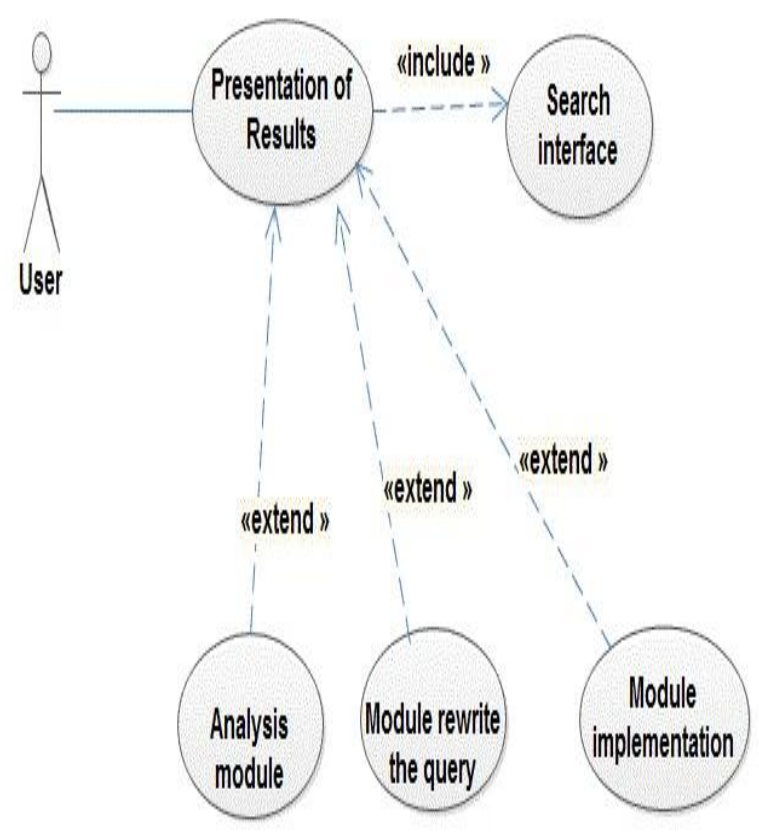

Fig 3. Use case diagram

In this section, we present a detailed description of the use cases more important, these use cases are typical.

Description of the use case "Presentation of results"

Table1. Summary of identification

\begin{tabular}{|l|l|}
\hline title & Presentation of results \\
\hline goal & $\begin{array}{l}\text { This use case provides an } \\
\text { opportunity for users to search } \\
\text { various data sources. }\end{array}$ \\
\hline abstract & Search for a value. \\
\hline actor & user \\
\hline
\end{tabular}

Table2. Description scenarios

\begin{tabular}{|l|l|}
\hline precondition & Search Interface \\
\hline sequence & $\begin{array}{l}\text { This use case begins when } \\
\text { the user wants to look at a } \\
\text { value in the data sources. }\end{array}$ \\
\hline main scenario & $\begin{array}{l}\text { The user types the search } \\
\text { value, and the system } \\
\text { displays the search results } \\
\text { from different data sources. }\end{array}$ \\
\hline post provided & $\begin{array}{l}\text { Connect data sources using } \\
\text { ontological mediator. }\end{array}$ \\
\hline
\end{tabular}

Description of the use case "implementation module"

Table3. Summary of identification:

\begin{tabular}{|l|l|}
\hline title & Module implementation \\
\hline goal & $\begin{array}{l}\text { This use case allows you to } \\
\text { implement data sources. }\end{array}$ \\
\hline abstract & Connect to data sources. \\
\hline actor & Users. \\
\hline
\end{tabular}


Table4. Description of scenarios:

\begin{tabular}{|l|l|}
\hline precondition & $\begin{array}{l}\text { Search Interface, } \\
\text { presentation of results }\end{array}$ \\
\hline sequence & $\begin{array}{l}\text { This use case begins when } \\
\text { the user starts searching and } \\
\text { connects to data sources. }\end{array}$ \\
\hline main scenario & $\begin{array}{l}\text { The system displays the } \\
\text { search results from different } \\
\text { data sources. }\end{array}$ \\
\hline post provided & $\begin{array}{l}\text { Connect data sources using } \\
\text { ontological mediator. }\end{array}$ \\
\hline
\end{tabular}

Description of the use case "Rewriting the query"

Table5. Summary of identification:

\begin{tabular}{|l|l|}
\hline title & Rewriting the query \\
\hline goal & $\begin{array}{l}\text { decompose the query into sub } \\
\text { queries. }\end{array}$ \\
\hline abstract & $\begin{array}{l}\text { express the query generated } \\
\text { initially. }\end{array}$ \\
\hline actor & Users. \\
\hline
\end{tabular}

Table6. Description of scenarios:

\begin{tabular}{|c|c|}
\hline precondition & $\begin{array}{l}\text { Search Interface, } \\
\text { presentation of results }\end{array}$ \\
\hline sequence & $\begin{array}{l}\text { This use case begins when } \\
\text { the user starts searching and } \\
\text { connects to data sources. }\end{array}$ \\
\hline scenario & $\begin{array}{l}\text { The system displays the } \\
\text { search results from different } \\
\text { data sources. }\end{array}$ \\
\hline post provided & $\begin{array}{l}\text { Connect data sources using } \\
\text { ontological mediator. }\end{array}$ \\
\hline
\end{tabular}

Description of the use case "analysis module"

Table7. Summary of identification:

\begin{tabular}{|l|l|}
\hline Title & Analysis module \\
\hline goal & $\begin{array}{l}\text { Parses the query in order to } \\
\text { detect any errors. }\end{array}$ \\
\hline abstract & $\begin{array}{l}\text { Analyze the generated query } \\
\text { initially. }\end{array}$ \\
\hline actor & Users. \\
\hline
\end{tabular}

Table8. Description of scenarios

\begin{tabular}{|l|l|}
\hline precondition & $\begin{array}{l}\text { Search Interface, } \\
\text { presentation of results }\end{array}$ \\
\hline sequence & $\begin{array}{l}\text { This use case begins when } \\
\text { the user starts searching } \\
\text { and connects to data } \\
\text { sources. }\end{array}$ \\
\hline main scenario & $\begin{array}{l}\text { The system displays the } \\
\text { search results from }\end{array}$ \\
\hline
\end{tabular}

\begin{tabular}{|l|l|}
\hline & different data sources. \\
\hline post provided & $\begin{array}{l}\text { Connect data sources } \\
\text { using ontological } \\
\text { mediator. }\end{array}$ \\
\hline
\end{tabular}

\subsubsection{Sequence diagram Launch Research}

The figure below shows the sequence diagram of launching a search:

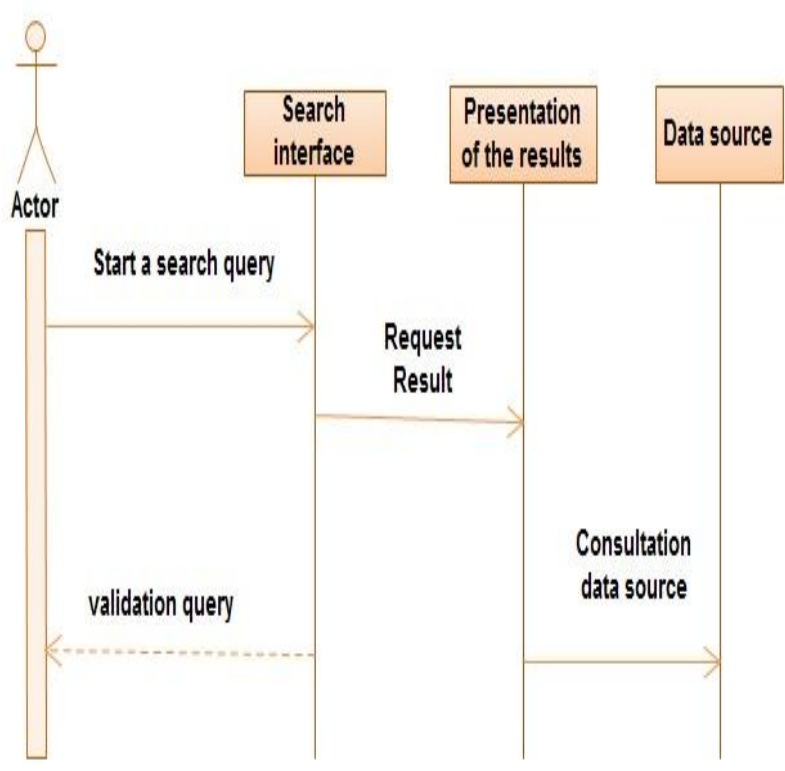

Fig 4. Sequence diagram Launch Research (1)

To get a result, the user Launches a search query, and then specify the language he wants and then validate their choice.

\subsubsection{Sequence diagram presentation of results}

The figure below shows the sequence diagram of the presentation of the results is as follows:

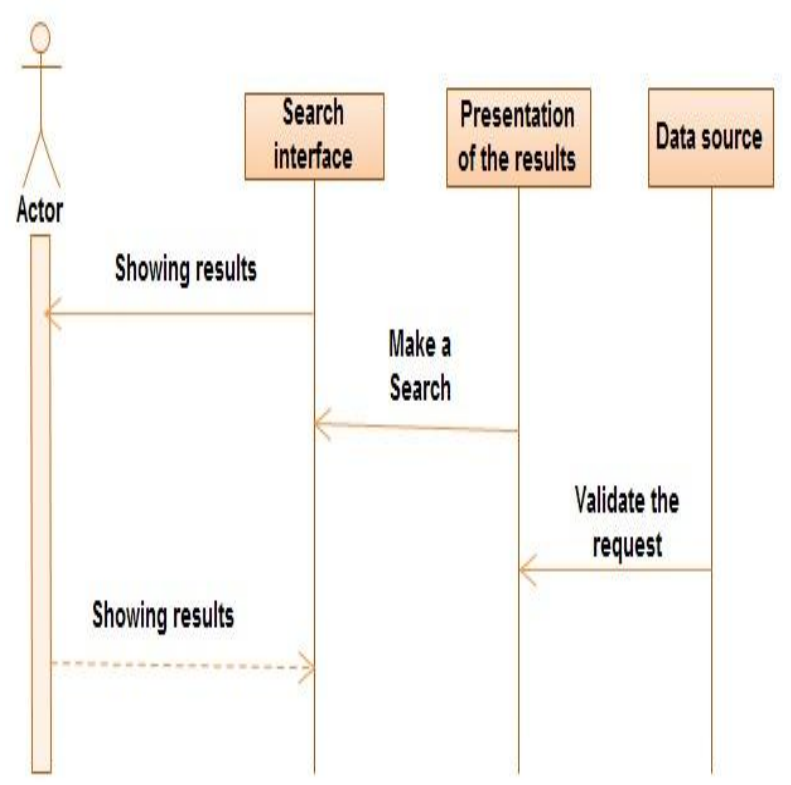

Fig 5. Sequence diagram presentation of results (2) 
The user requests the list of results support the type of language chosen, the system lists the results support the semantic correspondence.

\subsubsection{Class diagram}

The figure below shows the class diagram which includes the classes involved in the mediation system:

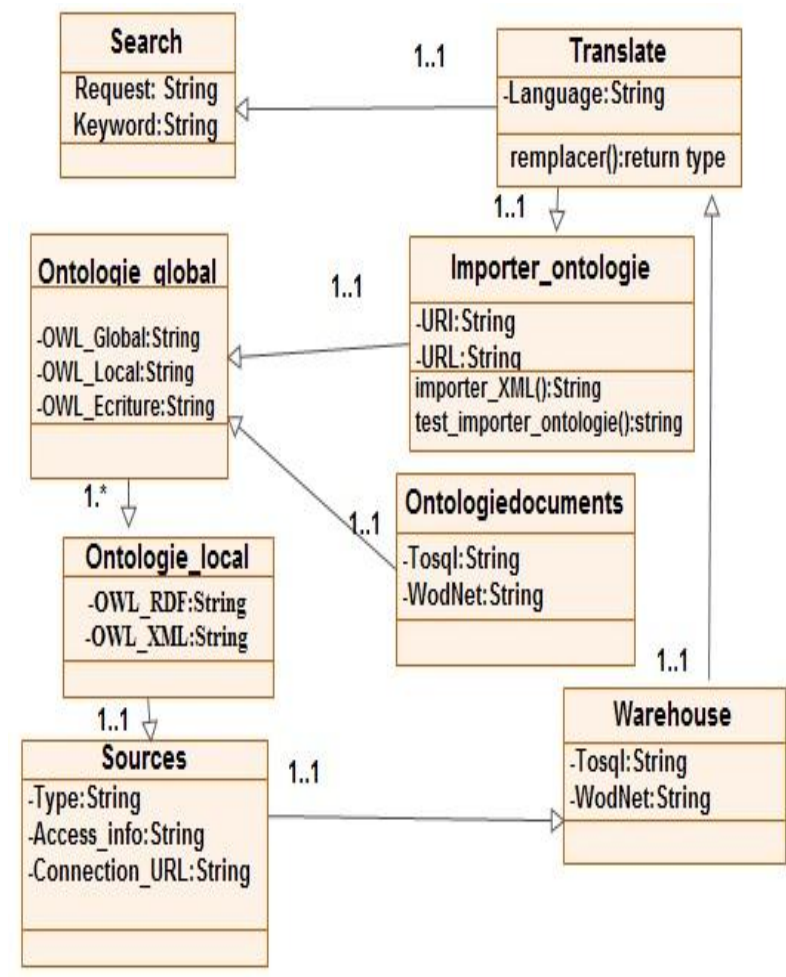

Fig 6. Class diagram

Table 9: list of classes and description

\begin{tabular}{|l|l|}
\hline Classe & Description \\
\hline search & $\begin{array}{l}\text { Brings together information } \\
\text { on research }\end{array}$ \\
\hline Translate & $\begin{array}{l}\text { Presents all the information } \\
\text { about the translation }\end{array}$ \\
\hline Ontologie_Global & $\begin{array}{l}\text { Includes all categories of } \\
\text { parameters for each } \\
\text { correspondence }\end{array}$ \\
\hline Importer_ontologie & $\begin{array}{l}\text { Includes all categories of } \\
\text { parameters to import } \\
\text { Ontology }\end{array}$ \\
\hline Ontologiedocuments & $\begin{array}{l}\text { Includes all objects related } \\
\text { documents for each } \\
\text { ontology. }\end{array}$ \\
\hline Warehouse & $\begin{array}{l}\text { Presents all the information } \\
\text { about a local ontology }\end{array}$ \\
\hline Sources & $\begin{array}{l}\text { Presents all the information } \\
\text { about a warehouse }\end{array}$ \\
\hline
\end{tabular}

\subsection{Modeling with Protege}

In the context of our modeling tool we have chosen to create the ontology is none other than Protege2000 (Protégé200, 2008) since it allows visualization and manipulation comfortable and intuitive concepts and relationships compose the ontology. It also consists of a Java library that can be extended to create real applications for knowledge bases using an inference engine for reasoning. Protege2000 can also program or import a large number of "plugins" which can be downloaded directly from its official website. These plugins add new features such as the ability to edit the ontology with different formats for describing ontology (RDF (S), OIL, DAML + OIL, OWL) [21].

\subsubsection{Conceptualization}

This step is the most important because it will generate the skeleton of our ontology we have for example to show the mapping, conceptual vocabulary between the community of teachers and students.

It involves the use of different data sources that exist in the field of tourism to keep only the concepts that constitute the hierarchy of the ontology.

After collecting the necessary information, the next step was to create classes and concepts to form the structure of the ontology we have for example vocabulary shared between the community of teachers and students.

The strategy we have adopted in the hierarchy from the most general concepts to more specific concepts. The study, analysis and classification of concepts collected from these different sources of data allowed us to share in 9 major super classes.

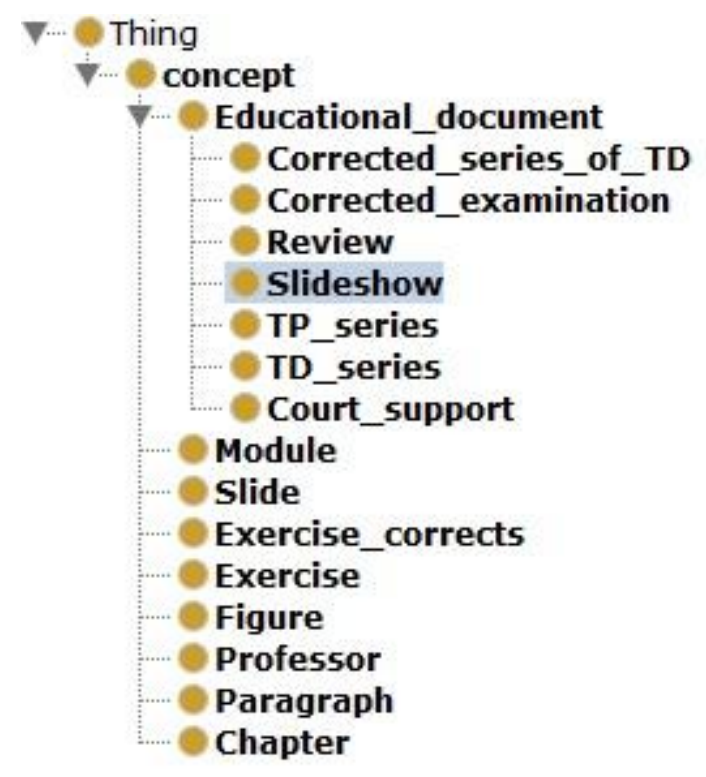

Fig 7. Class hierarchy "Virtual Library"

\subsubsection{Some examples of classes are:}

\section{A. Concept}

The class Concept is one of the main classes. It is the class that collects the individual. 


\begin{tabular}{|l|}
\hline http://www.owl-ontologies.com/Afoaf.owl\#Concept \\
\hline sub-class of: \\
http://www.owl-ontologies.com/Afoaf.owl\#Thing \\
\hline
\end{tabular}

In OWL DL this will be expressed with the following syntax: <!--http://www.owl-ontologies.com/Afoaf.owl\# concept --> <owl:Class rdf:about="\&Afoaf; concept ">

$</ o w l: C l a s s>$

\section{B. isauthor}

isauthor this property connects indicates this relation betwen Professor class and Educational_document class, and A teacher is an author of a educational document, if and only if it is he who wrote it.

http://www.owl-ontologies.com/Afoaf.owl\#isauthor

domain:

http://www.owl-ontologies.com/foaf.owl\#Professor

range:

http://www.owl-ontologies.com/foaf.owl\#

Educational_document

In OWL DL this will be expressed with the following syntax for class Professor: <!-- http://www.owlontologies.com/Afoaf.owl\# Educational_document -->

<owl:Class rdf:about="\&Afoaf; Educational_document "> $</$ owl:Class $>$

\section{Educational document}

This class represents the document that study in a university as an academic i.e. a Support during, a TD Series, slideshow, review, Corrected examination, Corrected series TD etc. Educational document is a subclass of the class Concept.

\begin{tabular}{|l|}
\hline $\begin{array}{l}\text { http://www.owl- } \\
\text { ontologies.com/Afoaf.owl\#Educational_document }\end{array}$ \\
\hline sub-class of: \\
http://www.owl-ontologies.com/Afoaf.owl\#Thing \\
\hline $\begin{array}{l}\text { sub-class of: } \\
\text { http://www.owl-ontologies.com/Afoaf.owl\#Concept }\end{array}$ \\
\hline $\begin{array}{l}\text { one of: \{ Support during, TD Series, slideshow, review, } \\
\text { Corrected examination, Corrected series TD \} }\end{array}$ \\
\hline
\end{tabular}

\section{D. teach}

A professor teaches a module during an academic year, and if only if it is responsible for courses and when he writes documents educational on this module

\begin{tabular}{|l|}
\hline http://www.owl-ontologies.com/Afoaf.owl\# teach \\
\hline domain: \\
http://www.owl-ontologies.com/foaf.owl\#Professor \\
\hline range: \\
http://www.owl-ontologies.com/foaf.owl\# Module \\
\hline E. provides
\end{tabular}

An educational document provides a module if and only if it is written (a was written) by (was) the teacher of this module, and when using it (had used) to teach this module its students.

http://www.owl-ontologies.com/Afoaf.owl\# provides

domain:

http://www.owl-ontologies.com/foaf.owl\#

Educational document

range:

http://www.owl-ontologies.com/foaf.owl\# Module

\subsubsection{List of property}

In the following table we will present property for each concept, and has inherited property of a concept father. 

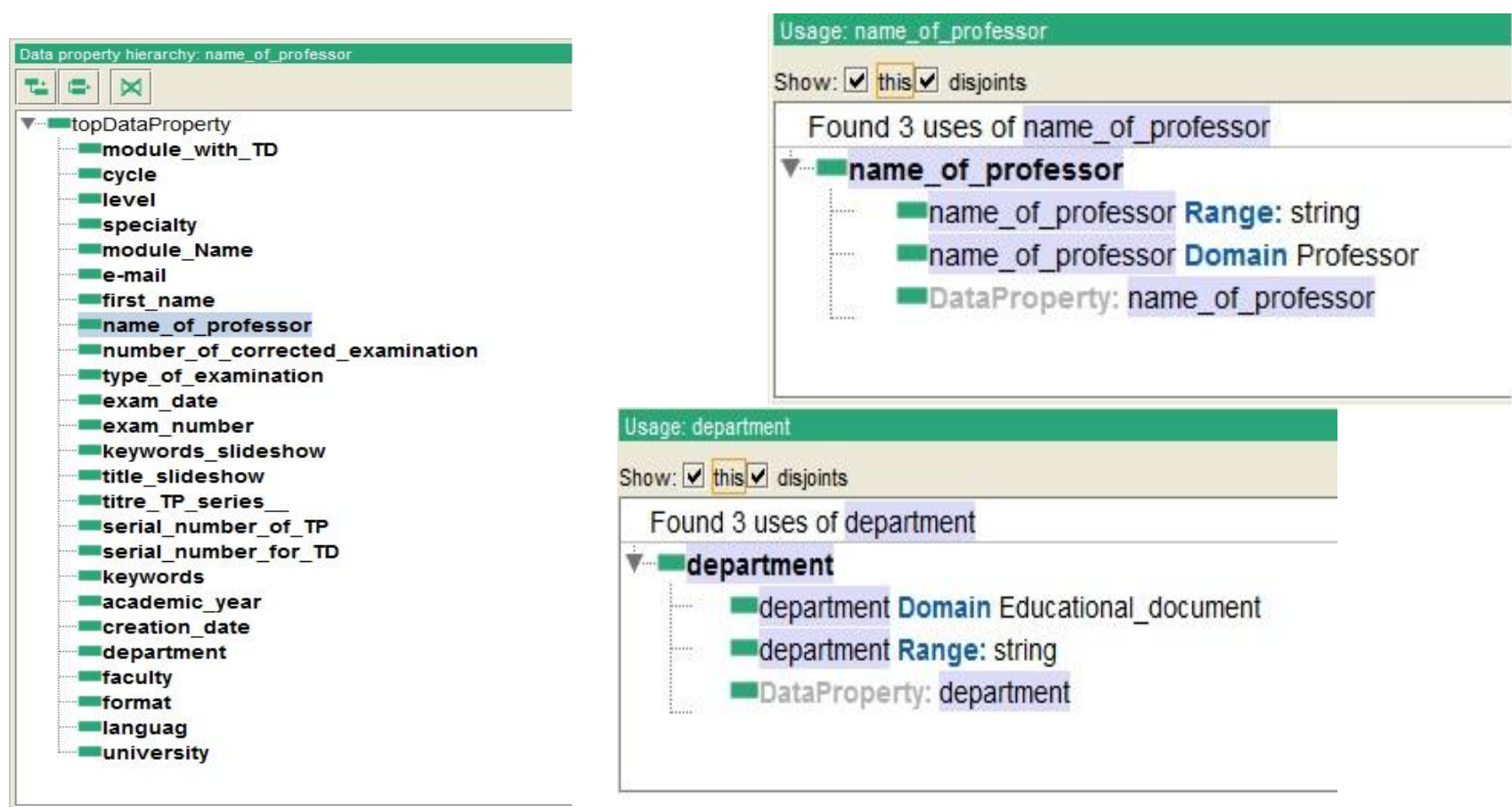

Fig8. Extract the Data property defined in the ontology

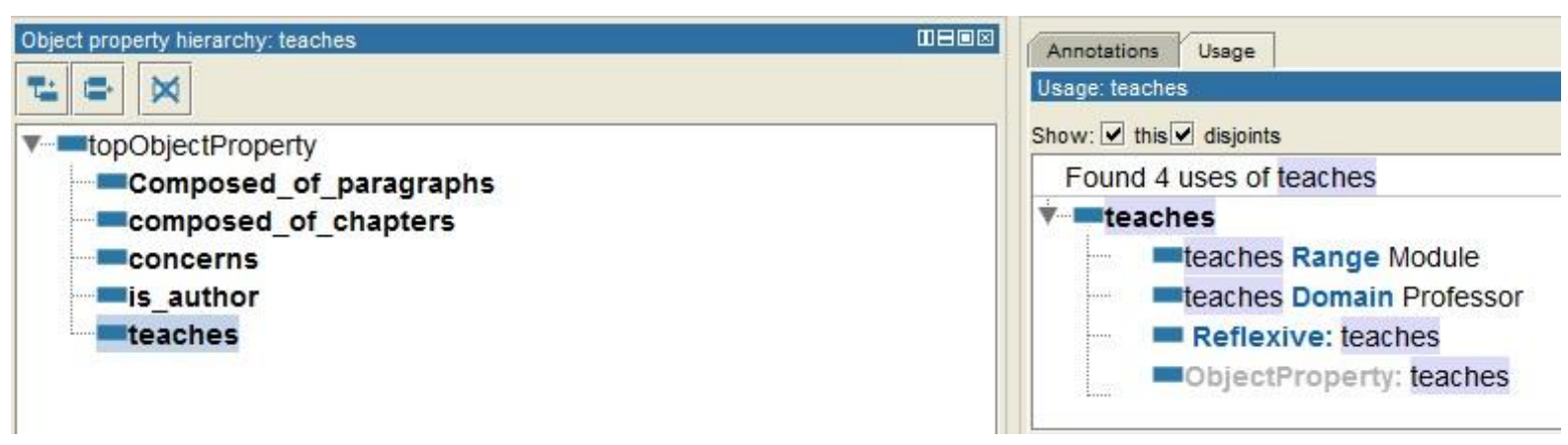

Fig9. Extract the Object property defined in the ontology

We also added annotations to enrich our ontology (Figure,). Finally, after having defined the class hierarchy, the next step was to assign properties to concepts. Ontology defines both properties of objects and properties (Figure,).

\section{- $\quad$ Verification}

The compliance and consistency of our ontology was performed using the FACT ++ reasoned protege 2000 available. Applying this reasoning to detect the slightest anomalies and inconsistencies in the hierarchy of the ontology. We can also say that our ontology is valid because the semantics we have expressed is restricted to the field of libraries.

$$
\text { - Use }
$$

This phase includes all activities based more or less directly to the layoff of ontology, annotation of resources or query resolution. In the current state, the knowledge base must be integrated into the ontology. In this stage of our development, we added instances manually to test the system. These bodies offer us the opportunity to conduct research guided by this ontology.

Bodies added information concerning some basic examples of libraries.

\section{CONCLUSION}

In this paper we presented the various phases through which we had to make the design of the ontology, where we followed the method ontologies, and presented the results of the design in the form of a list of concepts a list of relationships and other attributes of the protected software in addition to a hierarchical representation and another in the form of a UML diagram, and to present the features of each of the players in our application we used a diagram use case.

This article crown design phase, in which we presented the different diagrams to identify the various diagrams that reflect the functional aspect of the module, ie the use case diagrams, sequence diagrams and class diagrams.

Then we showed the different steps we followed for system modeling using the Protégé editor.

The next step is to make this ontology operational is usable by a computer, in addition to the detail of the application.

\section{ACKNOWLEDGMENTS}

Published research in this paper was partly doing doctoral research at the Faculty of Informatics, University, Faculty of Science. University Abdelmalek Essaadi. 


\section{REFERENCES}

[1] N. Noy, M. Musen.: The PROMPT suite: interactive tools for ontology merging and mapping. International Journal of Human-Computer Studies, 59(6): pp 983-1024,2003.

[2] G.Stumme, A. Madche.: FCA-Merge: Bottomup merging of ontologies. In Proc. 17thInternational Joint Conference on Artificial Intelligence (IJCAI), pp 225-234, Seattle (WA US), 2001.

[3] Y. Kalfoglou, M. Schorlemmer.: Ontology mapping: the state of the art. The Knowledge Engineering Review, 18(1):1-31, 2003.

[4] Laïla Benhlima and Dalila Chiadmi. «Vers l'interopérabilité des systèmes d'information hétérogènes». e-TI - Electronic Journal of Information Technology, Number 3, 27 December 2006, http://www.revue-eti.netdocument.php?id=1166.

[5] 10 February 2004, RDF is a standard model for data interchange on the Web. In ligne : http://www.w3.org/RDF/

[6] T.R.Gruber.A translation approach to portable ontologies. Knowledge A cquisition 5(2):199-220,1993.

[7] Using explicit ontologies in KBS development - Heijst, Schreiber, and al. - 1997.

[8] Fensel D., Ontologies: A Silver Bullet for knowledge Management and Electronic Commerce, Springer, Berlin-Heidelberg-New York, 2004.

[9] Sticef.org (2004) The role of ontological engineering in the field of ILE.

[10] Sticef.org (2003) Contribution of ontological engineering in distance learning environments

[11] Semantic Web Programming,John Hebeler,Matthew Fisher,Ryan Blace,Andrew Perez-Lopez,septembrie 2009.

[12] Tim Berners-Lee, James Hendler, Ora Lassila The Semantic Web, Scientific American, May 2001

[13] Jean Charlet, Laublet Philippe, Chantal Reynaud (2003) Specific action 32 CNRS / STIC: Semantic Web: Final Report

[14] Brickley, D., Guha, R. (2003). RDF vocabulary Description Language 1.0 RDF Schema. http://www.w3.org/TR/rdf-schema, W3C Working group

[15] Connolly, D. F. Van Harmelen, Horrocks, I, Mc-Guniess, D.L, Stein, L.A (2001). DAML+OIL reference Description. http://www.w3.org/TR/daml+oil reference

[16] Beech D., Malhotra A. And al. (1999). A formal Data Model and Algebra for XML.

[17] Fernandeze M., Simenon J. and Walder P. (2001). A semi-Monad for semi-structured Data. In International Conference on Database Theory.

[18] Wadjinny, F., Chiadmi, D. (2005) Taxonomy of XML algebra, Wotic

[19] Galanis L. et al. (2001). Following the Paths of XML Data: An Algebraic Framework for XML Query Evaluation. Technical Report.
[20] Zhang X. and Rundensteiner E.A. (2002.XAT). XML Algebra for the Rainbow System. Technical report WPICS-TR-02-24.

[21] Encyclopedia of Database Systems, P 963, ISBN 978-0387-39940-9, Copyright Holder Springer US. 2009.

[22] Semantic Web Technologies ,trends and research in ontology- based systems,John Davies, BT, UK,Rudi Studer,University of Karlsruhe,Germany,Paul Warren,BT, UK.

[23] Ouksel, A. 1992 Semantic Mechanisms for Cooperation in Heterogeneous Database Systems. International IEEE Conference on Man and Cybernetics, October.

[24] Ouksel, A., Iqbal A. 1999 Ontologies are not the Panacea in Data Integration: A Flexible Coordinator to Mediate Context Construction. Distributed and Parallel Databases, 7, 1-29.

[25] Sheth, A. Changing Focus on Interoperability in Information Systems: from System, Syntax, Structure to Semantic. Kluwer Academic Publishers, 1999, pp. 5\{29. In M.F. Goodchild, M.J. Egenhofer, R. Fegeas, and C.A. Kotman, editors. Interoperating Geo-graphic Information Systems. Kluwer Academic Publishers, Norwell, MA, pages 5\{29, Ouksel, A. 1992 Semantic Mechanisms for Cooperation in Heterogeneous Database Systems. International IEEE Conference on Man and Cybernetics, October.

[26] Prud'Hommeaux, E., and Seaborne, A. SPARQL Query Language for RDF. Tech. rep, World Wide Web Consortium, January 2008

[27] Doan, AnHai, Madhavan, Jayant, Domingos, Pedro, Halevy, Alon.: Learning to map between ontologies on the Semantic Web. In www, pp 662-673, 2002.

[28] M. Ehrig.: Ontology alignment: bridging the semantic gap. Semantic web and beyond: computing for human experience. Springer, New-York (NY US), 2007.

[29] M. Ehrig, S. Staab.: QOM - quick ontology mapping. In van Harmelen et al, pp 683- 696, 2004

[30] J. Tang, J. Li, B. Liang, X. Huang, Y. Li, K. Wang.: Using Bayesian decision for ontology mapping. Journal of Web Semantics, 4(1): pp 243-262, 2006.

[31] P. Bouquet, L. Serafini, S. Zanobini.: Semantic coordination: A new approach and an application. In Proc. 2nd International Semantic Web Conference (ISWC), volume 2870 of Lecture notes in computer science, pp 130-145, Sanibel Island (FL US), 2003a.

[32] P. Bouquet, B. Magnini, L. Serafini, S. Zanobini.: A SAT-based algorithm for context matching. In Proc. 4th International and Interdisciplinary Conference on Modeling and Using Context (CONTEXT), volume 2680 of Lecture notes in computer science, pp 66-79, Stanford (CA US), 2003 b.

[33] Raji Ghawi , "Ontology-based cooperation of information systems : contributions to database-toontology mapping and XML-to-ontology mapping", Université de Bourgogne (15/03/2010), Nadine Cullot (Dir.),

2010 